\title{
COMMUNAL FIXED POINT IN FUZZY 2- METRIC SPACES
}

\section{${ }^{1}$ Venessa Sualog Casanova, ${ }^{2}$ Atianashie Miracle A, ${ }^{3}$ Eneji Samuel Eneji, ${ }^{4}$ Ibe Walter} Eyong \& ${ }^{5}$ Angib Maurice Udie

${ }^{1}$ Occidental Mindoro State College Main Campus, Labangan Poblacion, San Jose, Occidental Mindoro, Philippines.

${ }^{2}$ Department of Computer Science, Catholic University College of Ghana 3, 4,5 Department of Computer Science Federal College of Education Obudu Cross River State, Nigeria

\section{Copyrights}

Copyright for this article is retained by the author, with first publication rights granted to the journal. This is an open-access article distributed under the terms and conditions of the Creative Commons Attribution license (http://creativecommons.org/licenses/by/4.0/)

Received: October 22, 2021 Accepted: November 14, 2021 Published Online: November 17, 2021

\begin{abstract}
The objective of this paper is to emphasize the Common Fixed Point in Fuzzy 2- Metric Spaces and prove a common fixed point theorem of compatible mappings of type $(\mathrm{R})$ in fuzzy 2-metric space. We consider four mappings of which one is continuous. The results generalize many results in the literature. Some illustrative examples are furnished which demonstrate the validity of the hypotheses and degree of utility of our results.
\end{abstract}

Keyword: Compatible mappings, Common fixed point, Fuzzy Metric Spaces, Fuzzy 2-Metrc spaces, Fuzzy set.

\section{INTRODUCTION}

The notion of fuzzy sets was introduced initially by Zadeh (Chauhan et al., 2013). After that many authors have expansively developed the theory of fuzzy sets and applications Especially Deng [6]. Erceg [(Jhurree, 2005), Kramosil and Michalek (Muchiri et al., 2015). have introduced the concept of fuzzy metric spaces in different ways. (Kothari et al., 2014) the common fixed point theorems in fuzzy metric space, while Chauhan and Utreja (Davis et al., 1989) proved the common fixed point theorems in fuzzy 2-metric space. Rohen, Singh and Shambhu (Zhang et al., 2020) introduced the concept of compatible mappings of type (C) and later on it renamed as compatible mappings of type $(\mathrm{R})$.

Now we begin with some definitions.

Definition 1.1 : $*[0,1] \times[0,1] \rightarrow[0,1]$ is called a continuous $t$-norm, if $\left([0,1],{ }^{*}\right)$ is an abelian topological monoid. With unit 1 such that $a * b \leq c^{*} b$, whenever $a \leq \mathrm{c}$ and $c \leq d$ for all $a, b, c, d \in[0,1]$.

Definition 1.2 : The 3-tuple $(X, M, *)$ is called a fuzzy Metric space if $X$ is an arbitrary set, $*$ is a continuous $t$ norm and $M$ is a fuzzy set in $X^{2} \mathrm{x}[0, \infty]$, satisfying the following conditions $\forall x, y, z \in X$ and $t, s>0$

(i) $M(x, y, 0)=0$

(ii) $M(x, y, t)=1 \forall t>0$ if and only $=$

(iii) $M(x, y, t)=M(y, x, t)$

(iv) $M(x, y, t) * M(y, x, z) \leq M(x, z, t+s)$

$(\mathrm{v}) M(x, y),[0, \infty] \rightarrow(0,1)$ is left continuous.

Definition 1.3 : Let $*:[0,1] \times[0,1] \times[0,1] \rightarrow[0,1]$ be a binary operation and is said to be a continuous $t-$ norm if following conditions are satisfied 


\section{International Journal of Multidisciplinary Studies and Innovative Research \\ Publisher: Catholic University College of Ghana \\ ISSN: 2737-7172 (0), ISSN: 2737-7180 (P) \\ DOI:10.53075/ijmsirq543101 \\ DOI Url: http://doi.org/10.53075/ljmsirq543101}

$([0,1], *)$ is an abelian topological monood with unit 1and

$2 . \mathrm{x} 1 * \mathbf{y} 1 * \mathbf{z} 1 \leq \mathrm{x} 2 * \mathbf{y} 2 * \mathbf{z} 2$ whenever $\mathrm{x} 1 \leq \mathrm{x} 2, \mathrm{y} 1 \leq \mathrm{y} 2, \mathrm{z} 1 \leq \mathrm{z} 2$ for all $\mathrm{x} 1, \mathrm{y} 1, \mathrm{z} 1, \mathrm{x} 2, \mathrm{y} 2, \mathrm{z} 2$ in $[0,1]$

AMS Subject Classification: 47H10, 54H25

Definition 1.4: The 3-tuple $(X, M, *)$ is called a fuzzy 2-Metric space if $X$ is an arbitrary set, * is a continuous $t$-norm and $M$ is a fuzzy set in $X^{3} \mathrm{x}[0, \infty]$, satisfying the following conditions $\forall x, y, z \in X$ and $t, s>0$

(i) $M(x, y, \mathrm{z}, 0)=0$

(ii) $M(x, y, \mathrm{z}, t)=1 \forall t>0$ and when out of three points at least two points are equal,

(iii) $M(x, y, \mathrm{z}, t)=M(x, z, y, t)=M(y, z, x, t)$,

(iv) $M(x, y, \mathrm{z}, t 1 / t 2 / t 3) \geq M(x, \mathrm{y}, \mathrm{u}, t 1) * M(x, \mathrm{u}, z, t 2)) * M(\mathrm{u}, \mathrm{y}, z, t 3)$

(v) $M(x, y z),[0, \infty] \rightarrow(0,1)$ is left continuous.

Lemma 1.5 : For all $x, y \in X, M(x, y, \mathrm{z},$.$) is non-decreasing.$

Definition 1.6: Let $(X, M, *)$ be a fuzzy 2- Metric-space.

1. A sequence \{\} in $X$ is said to be convergent to a point $\in X$ (denoted by $\lim \quad=n \rightarrow \infty$

if $\lim M(, \mathrm{a})=,1 \forall \mathrm{a} \in \mathrm{X}$ and $t>0 n \rightarrow \infty$

2. A sequence $\{x n\}$ in $X$ is called a Cauchy Sequence if $\lim M(n+p, \quad n, \mathrm{a}, \quad t)=1, \forall \mathrm{a} \in \mathrm{X}, t>0$ and $p>0$ $n \rightarrow \infty$

3. A fuzzy 2- Metric-space in which every Cauchy sequence is convergent is said to be complete.

Lemma 1.7: Let $\{y n\}$ be a sequence in fuzzy 2-Metric-space $(X, M, *)$ If there exists a number $k \in$ $(0,1)$ Such that,

$M(y n+2, y n+1, k t) \leq M(y n+1, y n, t)$ for all $t>0$ and $n=1,2, \ldots$ Then $\{y n\}$ is a Cauchy sequence in $X$.

Lemma 1.8: If for all $x, y, \in X, t>0$ and for a number $k \in(0,1) M(,) \geq,(,,$,$) . Then out of three points$ at least two points are equal.

Definition 1.9: Let $A$ and $B$ be mappings from a fuzzy 2 -Metric-space $(X, M, *)$ into itself. The mapping $A$ and $B$ are said to be compatible, If

$\lim \mathrm{M}(A B x n, B A x n, \mathrm{z}, t)=1, \forall>0$

$n \rightarrow \infty$

whenever $\{x n\}$ is a sequence in $X$ such that $\lim A x n=\lim B x n=\mathrm{v}$, for some v $\in X$

$$
n \rightarrow \infty \quad n \rightarrow \infty
$$

Definition 1.10: Let $A$ and $B$ be mapping from a fuzzy 2-Metric-space $(X, M, *)$ into itself.

The mapping $A$ and $B$ are said to be compatible type (P) if

$\lim M(A A x n, B B x n, \mathrm{z}, t)=1 n \rightarrow \infty$

for all $t>0$, whenever $\{x n\}$ is sequence in $X$ such that $A x n=\lim B x n=\mathrm{v}$ for some v $\in X . n \rightarrow \infty$

Definition 1.11: Let $A$ and $B$ be mapping from a fuzzy 2-Metrc-space $(X, M, *)$ into itself. 


\section{International Journal of Multidisciplinary Studies and Innovative Research \\ Publisher: Catholic University College of Ghana \\ ISSN: 2737-7172 (O), ISSN: 2737-7180 (P) \\ DOI:10.53075/ijmsirq543101 \\ DOI Url: http://doi.org/10.53075//jmsirq543101}

The mapping $A$ and $B$ are said to be compatible of type (R) if, $\lim M(A B x n, B A x n, z, t)=1$, and $\lim M(A A x n, B B x n, \mathrm{z}, t)=1 n \rightarrow \infty \quad n \rightarrow \infty$ whenever $\{x n\}$ is a sequence in $X$, such that $\lim A x n=\lim B x n=\mathrm{v}$ for some $\mathrm{v} \in X$, and for all $t>$ $0 . n \rightarrow \infty \quad n \rightarrow \infty$

Proposition 1.12: Let $(X, M, *)$ be a fuzzy 2-Metrc space with $t * t \geq \in[0,1] \quad$, be contuous mappings from $\mathrm{X}$ taken itself. Then $A$ and $B$ are compatible if and only if they are compatible of type $(\mathrm{R})$.

Proposition 1.13: Let $(X, M, *)$ be a fuzzy 2-Metrc-space with $t * t \geq$ for all $t \in(0,1)$ and $A, B$ be the mapping from $X$ into itself, if $\mathrm{A}$ and $\mathrm{B}$ are compatible of type (R) and $A v=B v$ for some $\mathrm{v} \in X$, then $A B v=B B v=B A v=A A v$.

Proposition 1.14: Let $(X, M, *)$ be a fuzzy 2-Metric-space with $\mathrm{t} * \quad \geq \quad \in[0,1]$ and $A$ and $B$ be the mappings from $X$ into itself. If $A$ and $B$ are compatible of type (R) and $\{x n\}$ is a sequence in $X$. Such that lim $A x n=n \rightarrow \infty$

$\lim B x n=\mathrm{v}$ for some $\mathrm{v} \in X$ then, $n \rightarrow \infty$

(i) $\lim B A x n=A v$ if $A$ is continuous at v. $n \rightarrow \infty$

(ii) $A B v=B A v$ and $A v=B v$ if $A$ and $B$ are continuous at v.

\section{MAIN RESULTS}

In our main result we prove a common fixed point theorem for four mappings in fuzzy 2 -metric space. We prove the following:

Theorem 2.1: Let A, B, S and T is self-mappings on fuzzy 2- metric space (X, M, *) which satisfies:

(i) $\quad M(A x, B y, z, t) \leq \min \{M(A x, S x, z, t), M(S x, T y, z, t), M(A x, T x, z, t), M(S x, B x, z, t), M(T x, B x, z, t)$, $\mathrm{M}(\mathrm{Sy}, \mathrm{Tx}, \mathrm{z}, \mathrm{t})\}$

For all $\mathrm{x}, \mathrm{y} \mathrm{z} \in \mathrm{X}$ out of three points at least two is equl, and $\mathrm{t}>0$.

(ii) For every $\mathrm{x} 0$ in the sequence $\{\mathrm{xn}\}$ and $\{\mathrm{yn}\}$ in $\mathrm{X}$ and such that

$\mathrm{Ax} 2 \mathrm{n}=\mathrm{Tx} 2 \mathrm{n}+1=\mathrm{y} 2 \mathrm{n}+1$

$\mathrm{Bx} 2 \mathrm{n}+1=\mathrm{Sx} 2 \mathrm{n}+2=\mathrm{y} 2 \mathrm{n}+2, \mathrm{n}=1,2 \ldots$

(iii) Any subsequence of sequence $\{\operatorname{Txn}\}$ and $\{\operatorname{Sxn}\}$ convergent to any point $\mathrm{v}$ in $\mathrm{X}$,

(iv) One of $\mathrm{A}, \mathrm{B}, \mathrm{S}$ and $\mathrm{T}$ is continuous at $\mathrm{v}$,

(v) The pairs $(\mathrm{A}, \mathrm{S})$ and $(\mathrm{B}, \mathrm{T})$ are compatible of type $(\mathrm{R})$ then $\mathrm{v}$ is unique common fixed point of $\mathrm{A}, \mathrm{B}, \mathrm{S}$ and $\mathrm{T}$.

Proof: By putting $x=x 2 n$ and $y=x 2 n+1$ in (i), we write

$M(A x, B y, \mathrm{z}, t)=M(T x 2 n+1, S x 2 n, \mathrm{z}, t)$

$\geq \min \{M(T x 2 n+1, S x 2 n, \mathrm{z}, t), M(S x 2 n, T \times 2 n+1, \mathrm{z}, t)$,

$M(T \times 2 n, S x 2 n+1, \mathrm{z}, t), M(S \times 2 n+1, T \times 2 n, \mathrm{z}, t)\}$ 


\section{International Journal of Multidisciplinary Studies and Innovative Research \\ Publisher: Catholic University College of Ghana \\ ISSN: 2737-7172 (O), ISSN: 2737-7180 (P) \\ DOI:10.53075/ijmsirq543101 \\ DOI Url: http://doi.org/10.53075/ljmsirq543101}

Therefore $M(y 2 n+1, \mathrm{y} 2 n+2, \mathrm{z}, t) \geq \min \{M(y 2 n+1, y 2 n, \mathrm{z}, t), M(y 2 n, y 2 n+1, \mathrm{z}, t), M(y 2 n+1, y 2 n, \mathrm{z}, t), M(y 2 n$, $y 2 n+1, \mathrm{z}, t), M(y 2 n, y 2 n+1, \mathrm{z}, t), M(y 2 n, y 2 n, \mathrm{z}, t)\}$

Or $M(y 2 n+1, y 2 n+2, \mathrm{z}, t) \geq M(y 2 n, y 2 n+1, \mathrm{z}, t)$

Similarly

$M(y 2 n+2, y 2 n+3, \mathrm{z}, t) \geq M(y 2 n+1, y 2 n+1, \mathrm{z}, t)$

Therefore for $n=1,2,3 \ldots$

$M(y n+2, y n+1, \mathrm{z}, t) \geq M(y n+1, y n, \mathrm{z}, t)$,

So $\{y n\}$ is a Cauchy sequence and taking $n \rightarrow \infty$, we write

$A \times 2 n=T \times 2 n+1 \rightarrow \mathrm{V}$

$B \times 2 n+1=S x 2 n+1 \rightarrow \mathrm{v}$

Now suppose that $A$ is continuous. Since $A$ and $S$ are compatible type (R) and by proposition 1.12 we get $A A x 2 n$ and

$S A x 2 n \rightarrow A v$ as $n \rightarrow \infty$

Now putting $x=A x 2 n$ and $y=x 2 n+1$ in (i) we get

$M(A A x 2 n, B x 2 n+1, \mathrm{z}, t) \geq \min \{M(A A x 2 n, S A x 2 n, \mathrm{z}, t), M(S A x 2 n, T x 2 n+1, \mathrm{z}, t), \quad M(A A x 2 n, T T x 2 n+1, \mathrm{z}, t)$, $M(S A x 2 n, B T \times 2 n+1, z, t)$,

$M(T T x 2 n+1, B T x 2 n+1, \mathrm{z}, t), M(\operatorname{Sx} 2 n+1, \operatorname{TT} x 2 n+1, \mathrm{z}, t)\}$

Taking limit, we write

$M(A x, \mathrm{v}, z, t) \geq M(A v, v, z, t)$

Therefore $A v=\mathrm{v}$

Similarly by putting $x=S x 2 n$ and $y=x 2 n+1$, we write

$M(A S x 2 n, B x 2 n+1, \mathrm{z}, t) \geq \min \{M(A S x 2 n, S S x 2 n, \mathrm{z}, t), M(S S x 2 n, T x 2 n+1, \mathrm{z}, t), M(A S x 2 n, T B x 2 n-1, \mathrm{z}, t), M(S S x 2 n$, $B B \times 2 n+1, \mathrm{z}, t), M(T B \times 2 n-1, B B \times 2 n-1, \mathrm{z}, t), M(S \times 2 n+1, T B \times 2 n-1, \mathrm{z}, t)\}$

Taking the limit

$M(S v, \mathrm{v}, z, t) \geq M(S v, \mathrm{v}, z, t)$

Therefore

$\mathrm{Sv}=\mathrm{v}$

Similarly

$B v=T v=\mathrm{v}$.

Hence v is a common fixed points of $A, B, S$ and $T$.

For the uniqueness let $\mathrm{w}$ be another fixed point $A, B, S$ and $T$. Suppose $\mathrm{w} \neq \mathrm{v}$ then by (i) we see that $M(\mathrm{v}, \mathrm{w}, t) \geq$ $M(\mathrm{v}, \mathrm{w}, t)$

Giving a contradiction. Hence $\mathrm{w}=\mathrm{v}$.

This completes the proof of the theorem.

Theorem 2.2: Let $\left(X, M,{ }^{*}\right)$ be a complete fuzzy 2-Metric- space with $t^{*} t \geq t$ for all $t \in[0,1]$ and the condition. Let $A, B, S, T$ and $P$ be mapping from $X$ into itself such that

(i) $\quad P^{r}(X) \subset(A B)^{s}(X), P^{r}(X), P^{r}(X) \subset(S T)^{s}(X)$, 


\section{International Journal of Multidisciplinary Studies and Innovative Research \\ Publisher: Catholic University College of Ghana \\ ISSN: 2737-7172 (O), ISSN: 2737-7180 (P) \\ DOI:10.53075/ijmsirq543101 \\ DOI Url: http://doi.org/10.53075/ljmsirq543101}

(ii) There exists a number $k \in(0,1)$ such that

$M\left(P^{1} x, P^{1} y, \mathrm{z}, k t\right) \geq M\left((A B)^{s} x, P^{r} x, \mathrm{z}, t\right)^{*} M\left((S T)^{s} y, p^{r} y, \mathrm{z}, t\right)^{*} M\left((S T)^{s} y, P^{r} x, \mathrm{z}, t\right)$

$* M\left((A B)^{s} x, \quad y, \mathrm{z}, t(2-)\right) * M\left((A B)^{s} x,(S T)^{s} y, \mathrm{z}, t\right)$

For all $x, y, \in X$,out of three points at least two is equal, $\in(0,2)$ and $r, s, t>0$.

(iii) $\quad P B=B P, P T=T P, A B=B A, S T=T S$.

(iv) $\quad A$ and $B$ are continuous.

(v) The pair $\left\{,(A B)^{s}\right\}$ is compatible of type (R)

(vi) $\quad M\left(x,(S T)^{s} x, t\right) \geq M\left(x,(A B)^{s} x, t\right)$ for all $x \in X$ and $p, r, t>0$ then $A, B, S, T$ and $P$ have common fixed point in $X$.

Proof: By (i) since $P^{r}(X) \subset(A B)^{s}(X)$, for any $x 0 \in X$, there exists a point $x 1 \in \quad$ such that $\quad x 0=(A B)^{s} x 1$. Since ${ }^{1}(x) \subset(S T)^{s}(X)$, for this point $x 1$, we can choose a point $x 2 \in X$ such that ${ }_{1}=(\quad)_{2}$. Inductively, we can define a sequence $\{y n\}$ in $X$ as follows

$y 2 n=P^{r} \times 2 n=(A B)^{s} \times 2 n+1$

$y 2 n+1=P^{r} x 2 n=(S T)^{s} x 2 n+2$

For $n=0,1,2, \ldots$ by (ii), for all $t>0$ and $\quad=1-q$ with $\quad \in(0,1)$ we have

$M(y 2 \mathrm{n}+1, y 2 \mathrm{n}+2, \mathrm{z} ., k t)=M\left(P^{r} x 2 n+1, P^{r} x 2 n+2,, \mathrm{z} k t\right)$

$\geq \quad M\left((A B)^{s} x 2 n+1, P^{r} x 2 n+1, \mathrm{z}, t\right)^{*} M\left((S T)^{s} x 2 n+2, P^{r} x 2 n+2, \mathrm{z}, t\right)$

$* M\left((S T)^{s} \times 2 n+2, P^{r} x 2 n+1, \mathrm{z}, \quad t\right)^{*} M\left((A B)^{s} x 2 n+1, P^{r} x 2 n+2, \mathrm{z},(2-) t\right)$

*M((AB) $\left.)^{s} \times 2 n+1,(S T)^{s} \times 2 n+2, \mathrm{z}, t\right)$

$=M(y 2 n, y 2 n+1, \mathrm{z}, t) * M(y 2 n+1, y 2 n+2, \mathrm{z}, q t) * M(y 2 n+1, y 2 n+1, \mathrm{z}, t) * M(y 2 n, y 2 n+2, \mathrm{z}, q t)$

$* M(y 2 n, y 2 n+1, \mathrm{z}, t)$

$\geq M(y 2 n, y 2 n+1, \mathrm{z}, t) * M(y 2 n+1, y 2 n+2, \mathrm{z}, q t)^{*} M(y 2 n, y 2 n+1, \mathrm{z}, t)^{*} M(y 2 \mathrm{n}+1, y 2 \mathrm{n}+2, \mathrm{z}, q t)$

$* M(y 2 \mathrm{n}, y 2 \mathrm{n}+1, \mathrm{z}, t)$

$\geq M(y 2 n, y 2 n+1, \mathrm{z}, t) * M(y 2 n+1, y 2 n+2, \mathrm{z}, t) * M(y 2 n, y 2 n+1, \mathrm{z}, q t)$

(1) Since the $t$-norm * is continuous and $M(x, y, \mathrm{z},$.$) is left continuous, letting q \rightarrow 1$ in (1) we have $M(y 2 n+1, y 2 n+2, \mathrm{z}, k t) \geq M(y 2 n, y 2 n+1, \mathrm{z}, t)^{*} M(y 2 n+1, y 2 n+2, \mathrm{z}, t)$

(2) similarly, we have also

$M(y 2 n+2, y 2 n+3, \mathrm{z}, k t) \geq M(y 2 n+1, y 2 n+2, \mathrm{z}, t)^{*} M(y 2 n+2, y 2 n+3, \mathrm{z}, t)$

(3) Thus from (2) and (3), it follow that

$M(y n+1, y n+2, \mathrm{z}, k t) \geq M(y n, y n+1, \mathrm{z}, t)^{*} M(y n+1, y n+2, \mathrm{z}, t)$ for $n=1,2, \ldots .$. and so, for positive integers $n, p$

$M(y n+1, y n+2, \mathrm{z}, k t) \geq M(y n, y n+1, \mathrm{z}, t) * M(y n+1, y n+2, \mathrm{z}, \quad)$

Thus, since $M(y n+1, y n+z, z,(\quad)) \rightarrow 1$ as $p \rightarrow \infty$, we have

$M(y n+1, y n+2, \mathrm{z}, k t) \geq M(y n, y n+1, \mathrm{z}, t)$.

By lemma 1.7, $\{y n\}$ is a Cauchy sequence in $X$ and so, since $X$ is complete, $\{y n\}$ converges to a point $v \in$. Since 


\section{International Journal of Multidisciplinary Studies and Innovative Research \\ Publisher: Catholic University College of Ghana \\ ISSN: 2737-7172 (O), ISSN: 2737-7180 (P) \\ DOI:10.53075/ijmsirq543101 \\ DOI Url: http://doi.org/10.53075/ljmsirq543101}

$\left\{P^{r} x n\right\},\left\{(A B)^{s} x 2 n+1\right\}$ and $\left\{(S T)^{s} x 2 n+2\right\}$ are subsequences of $\{y n\}$, they also converge to the point $v$, that is, as $n \rightarrow \infty, P^{r} x n, P^{r} x 2 n+1,(S T)^{s} x 2 n+1 \rightarrow$ v. Since $A, B$ are continuous and the pair $\left\{P^{r},(\quad)\right\}$ is compatible of type $(\mathrm{R})$, by preposition 1.12 we have, as $n \rightarrow \infty$,

$P^{r}((\quad)) x 2 n+1 \rightarrow(\quad) \mathrm{v},(\quad)^{2} \times 2 n+1 \rightarrow(\quad) \mathrm{v}$

By (ii) with $=1$, we have

$M\left(P^{r}(\quad) x 2 \mathrm{n}+1, P^{r} x 2 n+2, \mathrm{z}, k t\right) \geq M\left((\quad)^{2 \mathrm{~s}} \times 2 n+1, P^{r}(\quad) x 2 n+2, \mathrm{z}, t\right)$

$* M\left((\quad) x 2 \mathrm{n}+2, P^{r} x 2 n+2, \mathrm{z}, t\right) * M\left((\quad) x 2 n+2, P^{r}(\quad) x 2 n+2, \mathrm{z}, t\right) * M\left((\quad)^{2} x 2 n+1, P^{r} x 2 n+2, \mathrm{z}, t * M((\quad) x 2 n+1\right.$, $\left.(S T)^{s} x 2 n+2, \mathrm{z}, t\right)$

which implies that as $n \rightarrow \infty$,

$M\left((A B)^{s}, \mathrm{v} . \mathrm{v}, \mathrm{z}, k t\right) \geq 1 * 1 * M\left(\mathrm{v},(A B)^{s} v, \mathrm{z}, t\right)^{*} M\left((A B)^{s} v \mathrm{v}, t\right)^{*} M\left((A B)^{s} v, \mathrm{v}, \mathrm{z}, t\right)$

$\geq \quad M\left((A B)^{s} v, \mathrm{v}, \mathrm{z}, t\right)$

Therefore, by lemma 1.8, we have $(A B)^{s} v=\mathrm{v}$.

By (vi), since $M\left(\mathrm{v},(S T)^{s} \mathrm{v}, \mathrm{z}, t\right) \geq M\left((A B)^{s} v, \mathrm{z}, t\right)=1$ for all $r, s, t>0$, we have also $(S T)^{s} v=\mathrm{v}$. Again by (ii) with $=1$

$M\left(P^{r}(A B)^{s} x 2 n+1, P^{r} v, \mathrm{z}, k t\right) \geq M\left((\quad)^{2} x 2 n+1, P^{r}(\quad) x 2 \mathrm{n}+1, \mathrm{z}, t\right)^{*} M\left((S T)^{s} v, P^{r} v, \mathrm{z}, t\right)$

${ }^{*} M\left((S T)^{s} v, P^{r}(A B)^{s} x 2 n+1, \mathrm{z}, t\right) * M\left((A B)^{2 s} x 2 n+1\right.$,

This implies that, as $\left.n \rightarrow \infty, \operatorname{Prv}, t)^{*} M\left((A B)^{2 s} x 2 n+1,(S T)^{s} v, \mathrm{z}, t\right)\right\} M\left((A B)^{s} v, P^{r} v, \mathrm{z}, k t\right) \geq 1 * 1 * 1 * M\left((A B)^{s} v, P^{r} v, \mathrm{z}\right.$, $t)^{* 1}$

$\geq \quad M\left((A B)^{s} v, P^{r} v, \mathrm{z}, t\right)$

And so, by lemma $1.8,(A B)^{s} v=P^{r} v$.

Now, we show that $B S v=\mathrm{v}$. In fact by (ii) with $\quad=1$ and (iii), we have

$M\left(B^{s} v, \mathrm{v}, z, k t\right)=M\left(B^{s} P^{r} v, P^{r} v, \mathrm{z}, k t\right)=M\left(P^{r} B^{s} v, P^{r} v, \mathrm{z}, k t\right)$

$\geq \quad\left\{M\left((A B)^{s}\left(B^{s} v\right), \quad P^{r} B^{s} v, \mathrm{z}, \quad t\right)^{*} M\left((S T)^{s} v, \quad P^{r} v, \mathrm{z}, \quad t\right)^{*} M\left((S T)^{s} v, \quad P^{r} B^{s} v, \mathrm{z}, \quad t\right) \quad * M\left((A B)^{s}\left(B^{s} v\right), \quad P^{r} v, \mathrm{z}\right.\right.$, $\left.t)^{*} M\left((A B)^{s}\left(B^{s} v\right),(S T)^{s} v, \mathrm{z}, t\right)\right\}$

$\geq \quad M\left(\mathrm{v}, B^{s} v, \mathrm{z}, t\right)$

By lemma 1.8 which implies that $B^{s} v=\mathrm{v}$. Since $\quad(A B)^{s} v=\mathrm{v}, A^{s} v=\mathrm{v}$.

Finally, we show that $T^{s} v=v$. Indeed by (ii) with $=1$ and (iii),

$M\left(T^{\mathrm{s}} v, \mathrm{v}, z, k t\right)=M\left(T s P^{r} v, P^{r} v, \mathrm{z}, k t\right)=M\left(P^{r} v, P^{r} T^{s} v, \mathrm{z}, k t\right)$

$\geq M\left((A B)^{s} v, P^{r} v, \mathrm{z}, t\right)^{*} M\left((S T)^{s}\left(T^{s} v\right), \operatorname{Pr}^{s} v, \mathrm{z}, t\right)^{*} M\left((S T)^{s}\left(T^{s} v\right), P^{r} v, \mathrm{z}, t\right)$

$* M\left((A B)^{s} v, \operatorname{Pr}^{s} v, \mathrm{z}, t\right)^{*} M\left((A B)^{s} v,(S T)^{s}\left(T^{s} v\right), \mathrm{z}, t\right)$

$=\quad 1^{*} 1^{*} M\left(T^{s} v, \mathrm{v}, z, t\right)^{*} M\left(\mathrm{v}, T^{\mathrm{s}} v, \mathrm{z}, t\right)^{*} M\left(\mathrm{v}, T^{\mathrm{s}} v, \mathrm{z}, t\right) \geq M\left(T^{s} v, \mathrm{v}, z, t\right)$

Which implies that?

$T^{\mathrm{s}} v=\mathrm{v}$

Since $(S T)^{s} v=\mathrm{v}$. We have $\mathrm{v}=(S T)^{s} v=S^{s} v$.

Therefore, by combining the above results, we have

$A^{s} v=B^{s} v=S^{s} v=T^{s} v=P^{r} v=\mathrm{v}$,

That is, $\mathrm{v}$ is the common fixed point of $A, B, S, T$ and $P$ for $r, s, t>0$.

The uniqueness of the common fixed point $z$ of $A, B, S, T$ and $P$ follows easily from (ii). This completes the proof. 


\section{International Journal of Multidisciplinary Studies and Innovative Research}

Publisher: Catholic University College of Ghana

ISSN: 2737-7172 (O), ISSN: 2737-7180 (P)

DOI:10.53075/ijmsirq543101

DOI Url: http://doi.org/10.53075/ljmsirq543101

\section{REFERENCES}

Chauhan, S., Khan, M. A., \& Sintunavarat, W. (2013). Common fixed point theorems in fuzzy metric spaces satisfying $\varphi$-contractive condition with common limit range property. Abstract and Applied Analysis, 2013. https://doi.org/10.1155/2013/735217

Davis, F. D., Bagozzi, R. P., \& Warshaw, P. R. (1989). User Acceptance of Computer Technology : a Comparison of Two Theoretical Models *. 35(8).

Jhurree, V. (2005). Technology integration in education in developing countries : Guidelines to policy makers 1. International Education Journal, 6(4), 467-483.

Kothari, C., Kumar, R., \& Uusitalo, O. (2014). Research Methodology. In New Age International. https://doi.org/http://196.29.172.66:8080/jspui/bitstream/123456789/2574/1/Research\%20Methodology.pd $\mathrm{f}$

Muchiri, J. M., Barchok, H. K., J, K. N., Education, F., \& Chuka, P. O. B. (2015). EFFECT OF COMPUTER assisted teaching strategy on students' achievement in agriculture in secondary schools in kenya. 3(8), 463-474.

Zhang, S., Lu, C., Jiang, S., Shan, L., \& Xiong, N. N. (2020). An Unmanned Intelligent Transportation Scheduling System for Open-Pit Mine Vehicles Based on 5G and Big Data. IEEE Access, 8(Figure 1), 135524-135539. https://doi.org/10.1109/ACCESS.2020.3011109 